\title{
Ostéopétrose ou maladie des os de marbre 1. Revue de la littérature
}

\section{Osteopetrosis or marble bone disease 1. Review of the literature}

\author{
YVES GERMANIER, JEAN-PIERRE CARREL, SEMAAN ABI NAJM, JACKY SAMSON
}

\section{RÉSUMÉ}

L'ostéopétrose est une maladie osseuse rare, due principalement à un dysfonctionnement des ostéoclastes, qui sont des cellules d'origine hématopoïétique, de la lignée macrophagique. Il existe différentes formes d'ostéopétrose qui ont une évolution variable allant du décès in utero à la forme restant totalement asymptomatique.

L'ostéopétrose résulte d'un défaut du remodelage qui aboutit à une hyperminéralisation du squelette entraînant une fragilité osseuse (fractures pathologiques souvent multiples), la compression des nerfs crâniens (atrophie optique...), des ostéomyélites des maxillaires et un étouffement de la moelle hématopoiétique pouvant évoluer vers une pancytopénie.

II n'y a pas de traitement satisfaisant pour l'ostéopétrose. Dans la forme maligne, la transplantation de moelle osseuse permet dans les cas favorables, de stopper l'évolution et de rétablir un remodelage osseux fonctionnel. (Med Buccale Chir Buccale 2006; 12: 135-45)

mots clés : ostéopétrose, fracture pathologique, compression nerveuse, ostéomyélites, pancytopénie

\section{SUMMARY}

Osteopetrosis is a rare disease due to a dysfunction of osteoclasts which are haematopoietic cells of macrophage lineage. There are different forms of osteopetrosis with a variable evolution from the death in utero to completely asymptomatic form.

Osteopetrosis results from a bone remodelling defect which leads to a hypermineralization of the skeleton with a bone weakness (pathological and often multiple fractures), multiple cranial nerves compression (optic atrophy...), osteomyelitis of the maxillas and pressure of haematopoietic marrow that can lead to pancytopenia.

There is no successfull treatment to osteopetrosis. In the malignant form transplantation of bone marrow can, in favourable cases, stop the evolution of the disease and restore a bone remodelling function. (Med Buccale Chir Buccale 2006; 12: 135-45)

key words : osteopetrosis, pathological fracture, nerve compression, osteomyelitis, panacytopenia 
médecine

buccale

chirurgie

buccale

VOL. $12, \mathrm{~N}^{\circ} 3$ 2006

page 136
L'ostéopétrose constitue un exemple de maladie osseuse rare, très intéressante car elle permet d'observer les nombreux effets liés à un trouble de la résorption osseuse par les ostéoclastes. On connaît leur rôle dans la formation, la croissance, le développement et le maintien du squelette au cours de la vie. Le dysfonctionnement des ostéoclastes se traduit par des perturbations primaires ou secondaires touchant de nombreux systèmes : le squelette et l'appareil locomoteur, l'œil, la cavité buccale, le système nerveux, l'hématopoïèse, ou le tractus gastro-intestinal.

Les mécanismes physiopathologiques impliqués dans l'ostéopétrose conduisent à une grande variété d'effets : insuffisance de l'hématopoïèse médullaire, de la croissance et du remodelage du squelette, de la régénération osseuse après une fracture, diminution de la taille des foramen osseux crâniens, troubles du métabolisme calcique, anomalies de l'odontogenèse, diminution de la vascularisation osseuse et troubles immunologiques prédisposant à l'infection.

L'ostéomyélite des maxillaires associée à l'ostéopétrose est rare et représente bien peu de choses dans la pathologie infectieuse de la cavité buccale. Les progrès réalisés dans la prise en charge de cette complication permettent néanmoins, en se référant à la physiopathologie, de proposer des mesures thérapeutiques, préventives et curatives, adaptées pour les autres ostéomyélites, en particulier celles liées aux affections ostéocondensantes.

\section{GÉNÉRALITÉS}

L'ostéopétrose, également connue sous le nom de maladie des os de marbre ou de maladie d'AlbersSchönberg, se traduit radiologiquement par une croissance et une sclérose osseuses excessives, avec un épaississement des corticales et un comblement, voire même une disparition complète des canaux médullaires. Malgré cet «os en excès», le squelette se révèle anormalement fragile et les os se fracturent facilement, à la façon d'un morceau de craie, pour des traumatismes mineurs. Cette hyperminéralisation des os aboutit à la compression des pédicules nourriciers, entraînant d'une diminution de la vascularisation et une augmenta- tion de la fréquence des infections osseuses, notamment dans la région maxillaire.

Le terme d'ostéopétrose (du grec osteon, os et petros, pierre) fut introduit par Karshner en 1926 afin de décrire une maladie héréditaire, caractérisée radiologiquement par une importante augmentation de la densité de l'os cortical et de son épaisseur aux dépens de la partie médullaire [1]. Le premier cas fut décrit brièvement (un paragraphe et aucune illustration) en 1904 par Heinrich Albers-Schönberg (1865-1921), un médecinradiologue allemand : il concernait un sujet de 26 ans présentant une fracture du fémur après avoir marché dans un nid-de-poule [2].

Dans les années 60, on distinguait deux formes cliniques : une forme autosomale dominante « bénigne ", fréquemment asymptomatique, et une forme autosomale récessive « maligne », généralement fatale durant les premières années de vie en l'absence de traitement [3]. Actuellement, plus de 500 cas d'ostéopétrose ont été rapportés dans la littérature ${ }^{[4]}$. La prévalence de la forme bénigne a récemment été estimée à près de 1:20 000 en Europe et de 1:100000 à 1:500000 en Amérique du Nord. La forme maligne est plus rare: elle est estimée à respectivement à 1:500000 aux EtatsUnis et à 1:200000 dans le reste du monde [3,5]. D'autres formes cliniques ont progressivement été décrites, montrant la complexité nosologique de la forme humaine. En 1972, trois publications différentes, provenant de l'Europe et des Etats-Unis, décrivent l'association d'une ostéopétrose avec une acidose tubulaire rénale ${ }^{[6-8]}$. En 1983, il a été démontré que cette forme résulte d'une dysfonction héréditaire du métabolisme, secondaire à un déficit en anhydrase carbonique II (AC II) [9,10].

Des cas d'ostéopétrose intermédiaire été ont décrits à la fin des années 70 [11]. Elle se manifeste dans l'enfance et comporte plusieurs signes de la forme maligne mais, à la différence de cette dernière, elle semble compatible avec une survie jusqu'à l'âge adulte. Son retentissement sur la longévité reste toutefois encore à déterminer [12,13]. En 1986, une forme d'ostéopétrose létale entraînant une mort néonatale précoce a été rapportée ${ }^{[14]}$ et, en 1987, Andersen et Bollerslev ont distingués radiologiquement deux types dans l'ostéopétrose bénigne ${ }^{[5]}$. 
L'association ostéopétrose sévère et maladie de stockage neuronal est signalée pour la première fois en $1988{ }^{[15]}$. Une forme transitoire infantile de la maladie survenant de manière sporadique in utero a été décrite au début des années 90 [16], de même que certaines formes post-infectieuses dont l'étiologie virale a été suspectée [16-18]. En 1995, un troisième type (" centrifuge ») a été proposé pour l'ostéopétrose bénigne [19]. Plus récemment, une forme iatrogène est observée chez un enfant recevant des bisphosphonates à fortes doses ${ }^{[20]}$. Enfin, diverses associations ont été rapportées : avec une maladie de Hirschprung ${ }^{[21]}$, une poïkilodermie [22] ou plus récemment le syndrome OL-EDA-ID associant ostéopétrose, lymphoedème, dysplasie ectodermique anhydrotique et immunodéficience ${ }^{[23]}$.

Chez l'homme on distingue 9 formes d'ostéopétrose en fonction de leurs caractéristiques cliniques, étiologiques et génétiques (Tab. 1) ${ }^{[3]}$.

\section{FORMES CLINIQUES}

\subsection{Ostéopétrose maligne}

Dans la plupart des cas, elle est diagnostiquée précocement dans l'enfance ${ }^{[4,24,25]}$. Elle se transmet selon un mode autosomal récessif mais elle s'exprime de manière variable dans une même fratrie, et il existe souvent chez les parents une consanguinité (jusqu'à $20 \%$ ) ${ }^{[3,4]}$.

Elle se révèle principalement de deux façons : par des fractures pathologiques de type transversal ou oblique, secondaires à la fragilité osseuse, ou par un retard du développement staturo-pondéral associé à de fréquentes infections des voies aérodigestives supérieures ${ }^{[25]}$. Parfois elle se révèle par une obstruction nasale due à une insuffisance du développement des sinus paranasaux [24]. Les fractures constituent une complication fréquente, même en l'absence de traumatismes importants. Dans la plupart des cas, elles sont peu déplacées et touchent soit la diaphyse ou la métaphyse des os longs, soit l'épiphyse. Les fractures épiphysaires sont difficiles à détecter, le retard mis pour le diagnostic et la difficulté de la contention chez l'enfant conduisent souvent à une réaction périostée exagérée avec néoformation osseuse corticale exubérante. Cette réaction peut être encore amplifiée par des saignements sous-périostés favorisés par la thrombopénie [25].

Les enfants atteints sont de petite taille, avec une déformation des métaphyses des genoux et des humérus en forme de massue (ou «Ehrlenmeyerflask deformity ») et/ou un élargissement du crâne médecine buccale chirurgie buccale

VOL. $12, \mathrm{~N}^{\circ} 3$ 2006

page 137

Tableau 1 : Différentes formes cliniques de l'ostéopétrose humaine ( $m$ : maladie ; AD : autosomal dominant ; AR : autosomal récessif) ${ }^{[3]}$.

Different clinical forms of human osteopetrosis (m: disease; AD autosomal dominant; AR: autosomal recessive).

\begin{tabular}{|l|c|}
\hline \multicolumn{1}{|c|}{ Formes } & Transmission \\
\hline Bénigne (adulte) & \\
\hline - type I & $\mathrm{AD}$ \\
\hline - type II (m. d'Albers-Schönberg) & $\mathrm{AD}$ \\
\hline - type III (centrifuge) & $?$ \\
\hline Maligne (enfant) & $\mathrm{AR}$ \\
\hline Déficit en AC II & $\mathrm{AR}$ \\
\hline Intermédiaire & $\mathrm{AR}$ \\
\hline Létale AR & \\
\hline Liée à une m. lysosomiale & $\mathrm{AR}$ \\
\hline Infantile transitoire & $?$ \\
\hline Post-infectieuse & - \\
\hline Autres formes & \\
\hline
\end{tabular}


médecine buccale chirurgie buccale

VOL. $12, \mathrm{~N}^{\circ} 3$ 2006

page 138 avec une proéminence frontale ${ }^{[4]}$. Ils peuvent présenter des lésions des nerfs crâniens par compression de ces nerfs dans les foramen crâniens : atrophie du nerf optique avec œdème papillaire, troubles oculo-moteurs (nystagmus), surdité ou paralysie faciale [3,4,26-29]. Plusieurs cas d'hydrocéphalie ont également été rapportés ${ }^{[28]}$. Les principaux signes cliniques de l'ostéopétrose maligne sont résumés dans le Tab. 2 .

Les os, en particulier les côtes, les vertèbres et les os longs sont très denses. Cette hyperminéralisation osseuse s'accompagne d'une oblitération presque complète des canaux médullaires. II en résulte une anémie et une neutropénie majeures, rapidement aggravées par l'hypersplénisme ; elles sont souvent responsables d'un décès précoce par infection ${ }^{[4,25]}$. Cette prédisposition particulière aux infections semble majorée par un déficit intrinsèque du système immunitaire, comme le suggère l'altération de la fonction granulocytaire et monocytaire parfois mise en évidence ${ }^{[30}$. L'ostéomyélite des maxillaires, qui constitue une complication fréquente de l'ostéopétrose, est traitée dans l'article qui est consacré aux manifestations stomatologiques de l'ostéopétrose.
L'hypersplénisme secondaire à l'hématopoïèse extramédullaire entraîne une thrombopénie suffisamment importante pour engendrer un trouble de

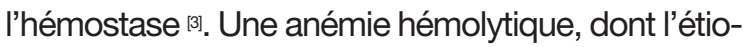
logie reste encore controversée, est fréquemment observée [24]. En l'absence de traitement, l'aggravation progressive des troubles hématologiques, responsable de complications sévères (anémie, hémorragies, pneumonie, septicémie...), entraîne le décès de la plupart des enfants atteints d'ostéopétrose maligne avant l'âge de 6 ans ${ }^{[4,24,25]}$.

Enfin, le rachitisme est une complication fréquente et d'intensité variable : paradoxalement, il existe un trouble de la minéralisation osseuse dans un contexte de bilan calcique global fortement positif [31-33. Sur le plan biochimique, on constate une hypocalcémie avec hypophosphatémie ainsi qu'une élévation des phosphatases acide et alcaline, de la PTH et du calcitriol. Cette forme particulière de rachitisme résulte de l'incapacité des ostéoclastes à maintenir une balance phosphocalcique adéquate dans le milieu extra-cellulaire ; il apparaît lorsque le taux sérique du calcium et des phosphates devient insuffisant pour assurer la minéralisation des tissus chondroïdes et ostéoïdes néoformés. II est favo-

Tableau 2 : Principales manifestations cliniques retrouvées dans 50 cas d'ostéopétrose maligne [29]. Principal clinical manifestations observed in 50 cases of malignant osteopetrosis [29].

\begin{tabular}{|l|l|}
\hline \multicolumn{1}{|c|}{ Signes cliniques } & Fréquence (\%) \\
\hline Atrophie optique & 78 \\
\hline Splénomégalie & 62 \\
\hline Hépatomégalie & 48 \\
\hline Retard staturo-pondéral & 36 \\
\hline Proéminence frontale & 34 \\
\hline Fractures & 28 \\
\hline Surdité & 22 \\
\hline Retard mental & 22 \\
\hline Elargissement du périmètre crânien & 22 \\
\hline Ostéomyélite (mandibule +++) & 18 \\
\hline Adénopathies & 18 \\
\hline Genu valgum & 16 \\
\hline Paralysie faciale & 10 \\
\hline Déformations thoraciques & 8 \\
\hline
\end{tabular}


risé par la réduction de l'apport calcique et par la corticothérapie généralement conseillées dans le traitement de l'ostéopétrose. II peut non seulement compromettre l'efficacité du traitement de l'ostéopétrose, mais il entraîne une diminution de la densité osseuse au contact des cartilages de croissance, simulant parfois une amélioration de l'ostéopétrose secondaire au traitement [33-37]. Les anomalies métaboliques et les principales complications de l'ostéopétrose maligne sont très nombreuses ; elles sont rappelées dans le schéma proposé par Reeves et coll. en $1981{ }^{[35]}$ (Fig. 1). Bien que les conceptions pathogéniques aient considérablement évolué - en particulier l'altération de la capacité de phagocytose ne constitue peut-être pas le principal facteur étiologique de l'ostéopétrose -, les mécanismes physiopathologiques proposés par Reeves et coll. demeurent néanmoins parfaitement valables, mais leur rôle respectif a changé.

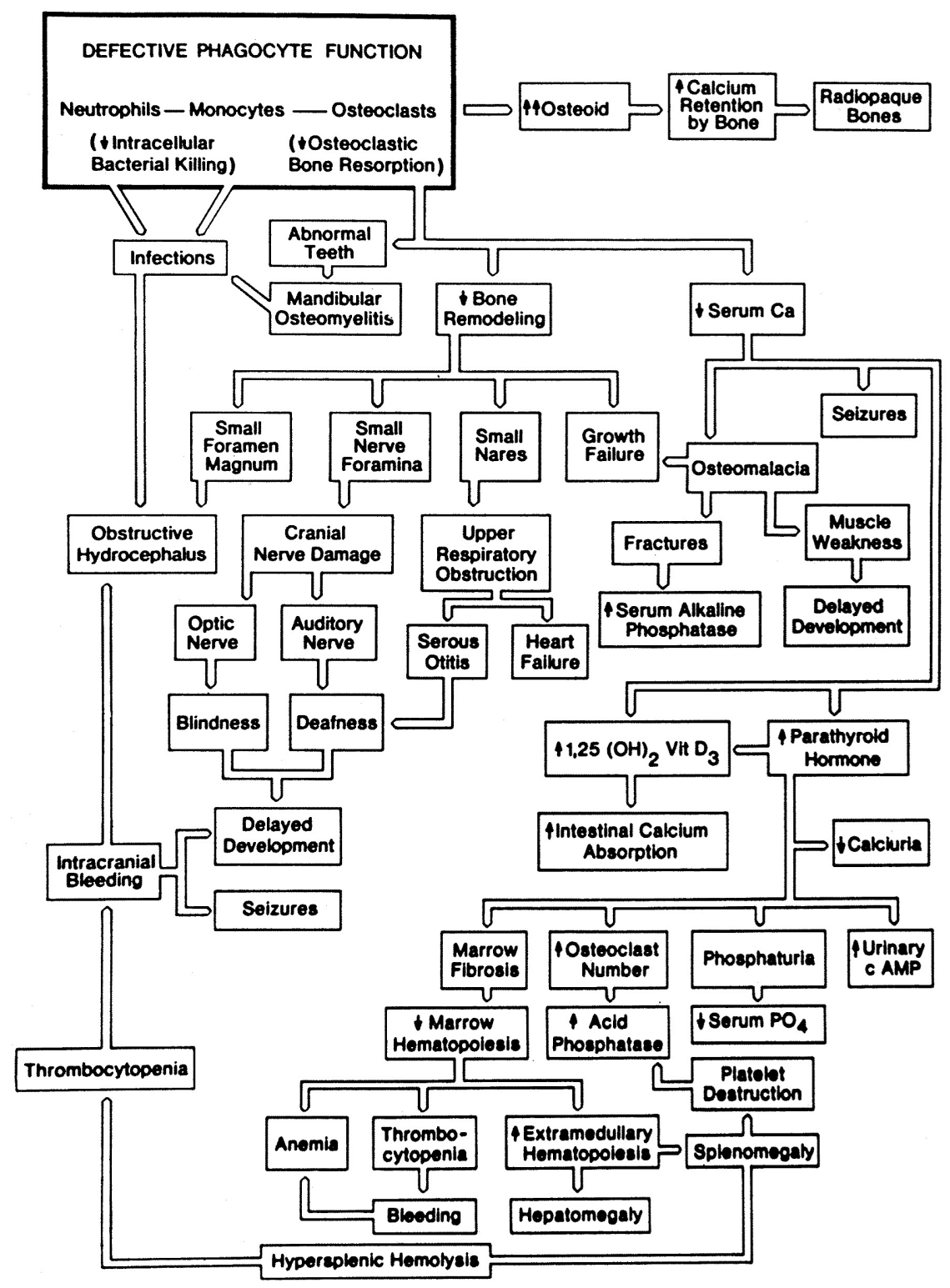

médecine

buccale chirurgie buccale

VOL. $12, N^{\circ} 3$ 2006 page 139

Figure 1 : Principales anomalies métaboliques et complications de l'ostéopétrose maligne (d'après Reeves et coll., 1985) [35].

Principal metabolic abnormalities and complications of malignant osteopetrosis (from Reeves and al. 1985) [35]. 
médecine buccale chirurgie buccale

VOL. $12, \mathrm{~N}^{\circ} 3$ 2006

page 140

\subsection{Ostéopétrose bénigne}

A l'opposé de la forme maligne, elle reste longtemps asymptomatique, les seules anomalies détectables étant radiologiques. Son diagnostic est souvent posé de manière fortuite pendant l'adolescence, à l'occasion d'une fracture due à un traumatisme mineur ou lors d'une infection [3]. En conséquence, la prévalence exacte de cette forme est très certainement sous-estimée, bien que de grandes familles comprenant de nombreux sujets atteints soient maintenant répertoriées [38]. La prévalence globale de la forme bénigne a été estimée à près de 1 cas pour 20000 habitants en Europe ${ }^{[5,39]}$ et 1 cas pour 100000 à 1 cas pour 500000 en Amérique du Nord ${ }^{[3]}$. Elle semble davantage toucher l'homme que la femme, peut-être parce que son activité physique l'expose plus aux traumatismes, ce qui faciliterait le diagnostic d'ostéopétrose [29]. La transmission se fait sur un mode autosomal dominant, avec une pénétration génétique variable : quelquefois, certains enfants de parents atteints sont indemnes ${ }^{[2,39}$. Le taux de pénétration est estimé à $75 \%{ }^{[29,40]}$; elle semble plus marquée dans la forme bénigne de type II [3].

Le diagnostic s'effectue à partir de l'aspect radiologique : ostéosclérose diffuse et progressive touchant essentiellement le squelette axial, avec une atteinte symétrique des os longs [38]. En 1987, Andersen et Bollerslev distinguent deux types sur la base de leur aspect radiologique ${ }^{[41]}$. Ces deux entités ont en commun une ostéosclérose généralisée intéressant en premier lieu le squelette axial. Le type I comporte une importante sclérose et une augmentation de l'épaisseur de la voûte crânienne, associées à une sclérose diffuse du rachis, sans épaississement des plateaux verté- braux, ni endostose («bone-within-a-bone ») dans les os du bassin. Le type II, qui représente en réalité la forme initialement décrite par AlbersSchönberg ${ }^{[2]}$, se caractérise par une sclérose osseuse prédominant à la base du crâne, un épaississement des plateaux vertébraux ("rugger-jersey spine") et une endostose intéressant le bassin. Les deux types se caractérisent en outre par un défaut de résorption de l'os endostal ; le remodelage de l'os trabéculaire est absent dans le type II [26,29,41]. En 1995, une troisième forme (type III) a été décrite : elle comporte une sclérose osseuse à développement «centrifuge », sans autres anomalies biochimiques ou histopathologiques ${ }^{[19]}$. En 1997, dans une famille norvégienne, on a pu observer un mélange de ces trois phénotypes confirmant la complexité nosologique de cette forme d'ostéopétrose.

Cliniquement, près de $45 \%$ des sujets atteints restent asymptomatiques quel que soit le type considéré $[5,29,42]$. Toutefois, si l'on examine l'ensemble de ces sujets de manière systématique, on constate que tous présentent des signes d'ostéopétrose déjà décelables dans l'enfance ${ }^{[43]}$. Les principales manifestations cliniques de l'ostéopétrose bénigne sont présentées dans le tableau 3 . Les fractures pathologiques constituent la manifestation révélatrice la plus fréquente de l'ostéopétrose bénigne : elles touchent entre 40 à $70 \%$ des sujets atteints ${ }^{[29,44]}$. Bien que des fractures multiples aient été décrites (jusqu'à 37 fractures chez un même patient), elles restent le plus souvent isolées et principalement localisées à la hanche et au fémur proximal. Dans la plupart des cas, elle sont traitées de manière conservatrice, même s'il y a un retard de consolidation [43,45,46]. L'extrême dureté de l'os rend toute ostéosynthèse

Tableau 3 : Principales manifestations cliniques observées dans 144 cas d'ostéopétrose bénigne [29,44]. Principal clinical manifestations observed in 144 cases of benign osteopetrosis ${ }^{[29,44]}$.

Signes cliniques

Patients asymptomatiques

Fractures

Douleurs osseuses

Atteinte des nerfs crâniens

Ostéomyélite (mandibule +++ )
Fréquence (\%)

\section{5}

$37-67$

20-25

$7-23$

7-10 
difficile à réaliser ${ }^{[25,45,46]}$. L'incidence des fractures semble plus élevée dans le type II ${ }^{[43]}$. Les douleurs osseuses, plus particulièrement dans la région lombaire, constituent un autre symptôme important, retrouvé dans $25 \%$ des cas [29,38].

L'atteinte des nerfs crâniens et l'ostéomyélite constituent deux autres complications spécifiques de cette maladie. La première touche jusqu'à $20 \%$ des sujets et elle est principalement due à la compression des nerfs optique, trijumeau, facial et vestibulo-cochléaire dans les foramen crâniens $[5,44,47,48]$. Elle varie selon le type et elle semble pouvoir s'atténuer avec l'âge [5,47-50]. L'ostéomyélite qui touche principalement la mandibule, représente une complication liée à la diminution de la vascularisation osseuse, secondaire à la compression des pédicules nourriciers par la sclérose ; elle peut être aussi favorisée par les troubles immunitaires souvent présents $[25,29,44]$.

Les examens physique et psychiatrique sont généralement normaux pour les deux types considérés. II en est de même pour l'espérance de vie ${ }^{55,44}$. On retrouve parfois associés : une arthrose, un syndrome du tunnel carpien, un hypogonadisme hypogonadotrophique [2,51,52] ; l'anémie par myélophtysie est rare ${ }^{[5,42]}$.

\subsection{Déficit en AC II}

Le déficit en AC II est une maladie métabolique congénitale, causée par diverses mutations du gêne AC2 [53], dont le rôle dans la pathogénie de

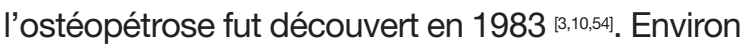
50 cas ont été rapportés dans la littérature ${ }^{\left[{ }^{[}\right] .} \mathrm{La}$ plupart touchent des sujets originaires de la région méditerranéenne et du Moyen-Orient car la consanguinité semble favoriser les mutations du gène AC2 dans de nombreux groupes eth-

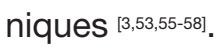

La maladie n'est en général pas découverte avant la grande enfance et associe, outre les signes classiques d'ostéopétrose, une acidose tubulaire rénale, des calcifications cérébrales, un retard mental d'importance variable, une petite taille, et des troubles de l'occlusion dentaire [9]. Les anomalies squelettiques ressemblent à celles observées dans les autres formes d'ostéopétrose mais, à la différence de celles-ci, elles peuvent régresser de manière spontanée au cours de l'évolution ${ }^{\left[{ }^{9} .\right.}$. L'acidose tubulaire rénale entraîne parfois l'apparition d'une apathie, d'une faiblesse musculaire et d'une paralysie transitoire d'origine hypokaliémique [56]. L'hématopoïèse extramédullaire pour compenser la myélophtysie est rare [59-61].

L'étude du déficit en $A C$ II revêt une importance particulière dans la mesure où elle fournit de nombreuses informations sur le rôle physiologique de cette enzyme dans les phénomènes de résorption osseuse. Les AC sont des métalloenzymes monomériques, ayant un poids moléculaire d'environ 29000 et le zinc comme cofacteur; on connait 4 formes chez l'homme (AC I, AC II, AC III et $A C$ IV). Elles jouent un rôle très important dans l'équilibre acido-basique : elles catalysent la transformation du $\mathrm{CO}_{2}$ et de l' $\mathrm{H}_{2} \mathrm{O}$ en $\mathrm{H}_{2} \mathrm{CO}_{3}$ qui se dissocie à son tour en $\mathrm{H}^{+}$et $\mathrm{HCO}_{3}{ }^{-[3,53,62,63]}$. L'AC II est l'isoenzyme qui possède le plus grand pouvoir catalytique dans la famille des AC. Elle est présente notamment dans les ostéoclastes où elle joue un rôle primordial dans les phénomènes de résorption osseuse [3,53,62,63]. II n'est donc pas surprenant qu'un déficit en AC II puisse se manifester par un trouble du remodelage osseux entraînant un épaississement généralisé du squelette. L'AC II est synthétisée à partir de la famille des gênes AC situés, chez l'homme, sur le bras long du chromosome 8 ; les gênes de l'AC II sont très largement distribués dans l'organisme ${ }^{[3]}$ : on les retrouve notamment dans le rein, le cerveau, les globules rouges, l'os, les monocytes périphériques et les macrophages ${ }^{[3,53,6,62,63]}$. Les AC semblent de plus jouer un rôle fondamental dans la synthèse des sécrétions du pancréas, de la salive, de l'humeur aqueuse, du liquide céphalo-rachidien et du suc gastrique ${ }^{[3,53,62,63] \text {. }}$

Les manifestations cliniques sont variables en fonction de l'origine géographique du sujet, ce qui suggère une hétérogénéité génétique ${ }^{[64]}$ : ainsi, les patients du Moyen-Orient présentent en général un retard mental et une acidose métabolique sévère mais relativement peu de fractures, les patients originaires d'Europe et d'Amérique du Nord font beaucoup plus de fractures ${ }^{[56,65]}$.

\subsection{Ostéopétrose intermédiaire}

Elle a une transmission autosomale récessive et se traduit par une petite taille, une macrocéphalie, une atteinte des nerfs crâniens, des fractures pathologiques récurrentes, des dents ankylosées médecine

buccale chirurgie buccale

VOL. $12, \mathrm{~N}^{\circ} 3$ 2006

page 141 
médecine

buccale

chirurgie

buccale

VOL. $12, \mathrm{~N}^{\circ} 3$ 2006

page 142 et des ostéomyélites des maxillaires. L'anémie est en général sévère. Le pronostic à long terme l'ostéopétrose intermédiaire reste à préciser [11-13,24].

\subsection{Ostéopétrose et maladie de stoc- kage neuronal}

Elle a une transmission autosomale récessive et possède les caractéristiques de la forme maligne, associées à des manifestations neurologiques : atrophie cérébrale généralisée et dilatation ventriculaire, responsables d'une hypertonie musculaire et de crises d'épilepsie récurrentes, atrophie optique, dégénérescence maculaire... Ces manifestations sont dues à une dégénérescence neuronale par accumulation de céroïdolipofuschine secondaire à un déficit de la fonction Iysosomiale [15,24]. Cette forme d'ostéopétrose est généralement fatale avant l'âge de 2 ans. Le traitement visant à stimuler la fonction ostéoclastique et la transplantation de moelle osseuse sont inefficaces ${ }^{[15,24]}$.

\subsection{Ostéopétrose létale}

Elle a une transmission autosomale récessive et provoque la mort in utero du fœtus. Elle peut être découverte pendant la grossesse : à l'échographie, elle se manifeste par une ostéosclérose généralisée, des fractures multiples et une hydrocéphalie ${ }^{[14,24]}$.

\subsection{Ostéopétrose post-infectieuse}

Des études en microscopie électronique ont révélé, au sein des ostéoclastes dans l'ostéopétrose bénigne, la présence d'inclusions virales ressemblant à la nucléocapside du paramyxovirus, ou des antigènes viraux provenant du virus de la rougeole ou du virus respiratoire syncytial [18]. II semble donc exister une forme post-infectieuse ${ }^{[66]}$. En 1990, on a découvert de façon sporadique, dans certaines cellules mononuclées de patients atteints d'une ostéopétrose bénigne, une reverse transcriptase comparable à celle de certains rétrovirus. L'expérimentation semble confirmer que des rétrovirus seraient capables de provoquer une ostéopétrose bénigne ou maligne $[17,18,66,67]$

Un trouble de la synthèse de facteurs impliqués dans la résorption osseuse - la PTH, l'interleukine-2, les superoxydes - a été évoqué pour expli- quer l'ostéopétrose post-infectieuse [3]. Pour mémoire, des structures ressemblant à des inclusions virales ont également été retrouvées dans d'autres maladies osseuses humaines [66,67].

\section{ASPECT RADIOLOGIQUE}

\subsection{Radiologie conventionnelle}

Le terme d'ostéopétrose a longtemps été utilisé dans la littérature de manière abusive pour décrire toute augmentation de la densité osseuse. De nombreuses études qui ont suivi la description originale de la maladie des os de marbre par Albers-Schönberg se basaient uniquement sur des critères radiologiques pour poser le diagnostic d'ostéopétrose : ainsi, d'autres maladies ostéosclérosantes comme les anomalies osseuses observées dans certaines leucémies, la myélofibrose, la fluorose... furent initialement regroupées sous cette entité ${ }^{[2,68]}$. Or l'ostéopétrose n'est pas la seule forme d'ostéosclérose, bien qu'elle soit certainement l'une des plus connues. Toutes les affections qui comportent une ostéocondensation, diffuse ou localisée, et qui peuvent être évoquées dans le diagnostic différentiel sont répertoriées dans le Tab. 4.

En radiologie conventionnelle, l'ostéopétrose se caractérise principalement par une augmentation généralisée et symétrique de la densité osseuse touchant à la fois la corticale (hyperostose) et la spongieuse (ostéosclérose) dont l'importance varie en fonction de la forme clinique considérée [3]. Dans les formes les plus avancées, elle aboutit à la disparition complète de la limite corticomédullaire, l'os présentant alors une densité corticale uniforme sur toute sa largeur. Dans les formes évoluées, la métaphyse des os longs est élargie en raison du trouble du remodelage osseux et elle prend une forme de massue ("Ehrlenmeyer-flask deformity"). Chez certains sujets, on observe parfois un phénomène d'endostose ("bone-within-a-bone") touchant principalement le tarse, les corps vertébraux, les phalanges des mains et des pieds et les ailes iliaques. Les régions métaphysaires et les ailes iliaques présentent souvent une alternance de bandes radio-transparentes et de bandes radioopaques, témoignant de l'évolution cyclique de 
Tableau 4 : Maladies comportant une ostéosclérose diffuse ou localisée [24].

Diseases with diffuse or localized osteosclerosis [24].

\section{MALADIES COMPORTANT UNE OSTÉOSCLÉROSE}

\section{Dysplasies osseuses}

Ostéosclérose autosomale dominante

Ostéosclérose centrale avec dysplasie ectodermique

Dysplasie crânio-diaphysaire

Dysplasie crânio-métaphysaire

Dysostosclérose

Hyperostose endostale

- hyperostose corticale généralisée

- sclérostéose

Dysplasie fronto-métaphysaire

Hyperostose corticale infantile (maladie de Caffey)

Nanisme hyperostotique de Lenz-Majewski

Mélorhéostose

Dysplasie métaphysaire (maladie de Pyle)

Dystrophie sclérosante mixte

Dysplasie occulo-dento-osseuse

Ostéodysplasie de Melnick et Needles

Ostéoectasie avec hyperphosphatasie

Ostéomésopycnose

Ostéopathie striée

Ostéopétroses

Ostéopoïkilose

Pachydermopériostose

Dysplasie diaphysaire progressive (maladie d'Engelmann)

Pycnodysostose

Sténose tubulaire (syndrome de Kenny-Caffey)

\section{Maladies métaboliques}

Déficit en anhydrase carbonique ॥

Fluorose

Intoxications: plomb, arsenic, béryllium, bismuth

Ostéosclérose associée au virus de l'hépatite C

Hypervitaminoses A et D

Hyper-, hypo- et pseudohypoparathyroïdie

Rachitisme hypophosphatasémique ou ostéomalacie

Syndrome du buveur de lait ("milk-alcali syndrome")

Ostéodystrophie rénale

Hypophosphatémie liée au chromosome X

\section{Autres}

Ostéomalacie axiale

Hyperostose squelettique diffuse idiopathique

Fibrogenèse osseuse imparfaite

Intoxication médicamenteuse intra-veineuse

Radiations ionisantes

Leucémies

Lymphomes

Mastocytose

Myélome multiple

Myélofibrose

Ostéomyélite

Ostéonécrose

Maladie de Paget

Maladie de Vaquez

Sarcoïdose

Anémie falciforme

Métastases osseuses ostéocondensantes

Sclérose tubéreuse de Bourneville la maladie, où des phases d'activité alternent avec des phases de rémission [24,25,68-70]. Les vertèbres ont un aspect typique sur les clichés de profil : image d'endostose avec un épaississement des plateaux réalisant l'aspect en sandwich. Le crâne est généralement épaissi et radioopaque. La mastoïde et les sinus paranasaux sont mal pneumatisés. Un épaississement et une radiotransparence des zones de croissance et des métaphyses peuvent s'observer chez les patients présentant un rachitisme associé $[24,46,71]$. Les caractères radiologiques de la forme bénigne sont hétérogènes et, dans la plupart des cas, il n'y a pas de troubles du remodelage osseux évidents $[11,24,41,46,47,70-72]$. Pour mémoire les deux types de la forme bénigne ont été individualisées par Andersen et Bollerslev sur la base de caractères radiologiques différents ${ }^{[4]}$. Le type I se caractérise par une importante sclérose et une augmentation de l'épaisseur de la voûte crânienne associées à une sclérose diffuse du rachis, mais sans épaississement des plateaux vertébraux, ni endostose touchant le bassin. Le type II comporte une sclérose osseuse prédominant à la base du crâne, un épaississement des plateaux vertébraux réalisant l'aspect en sandwich ("rugger-jersey spine") et une endostose touchant le bassin. Ces endostoses sont constituées d'os cortical dont l'origine exacte reste indéterminée ${ }^{[41]}$. Le type III se caractérise par une sclérose osseuse touchant surtout les os longs des membres ${ }^{[3]}$.

Le déficit en AC II se traduit par des anomalies radiologiques très similaires à celles des autres formes d'ostéopétrose, avec en plus des calcifications cérébrales. Ces dernières se développent vers l'âge de 5 ans et leur taille augmente progressivement pendant l'enfance, affectant presque exclusivement la substance grise corticale et les ganglions crâniens. Elles sont similaires, mais non identiques, à celles rencontrées dans la pseudo-hypoparathyroïdie et l'hypoparathyroïdie idiopathique ${ }^{[3,24,73]}$. Enfin, il semble que les anomalies du remodelage squelettique et les signes d'ostéopétrose rencontrés dans cette forme peuvent spontanément régresser avec l'âge [9]. Cette évolution paradoxale contraste avec l'aggravation de la sclérose osseuse proportionnelle à l'âge, généralement constatée dans les autres formes d'ostéopétrose ${ }^{[3,24]}$. médecine

buccale

chirurgie

buccale

VOL. $12, \mathrm{~N}^{\circ} 3$ 2006

page 143 


\subsection{Tomodensitométrie (CT-scan)}

Le CT-scan constitue le meilleur examen pour le diagnostic des compressions nerveuses dans les foramen crâniens. Cette méthode permet en effet une bonne évaluation de leur diamètre et se révèle particulièrement utile dans le suivi à long terme des patients, dans le contrôle post-opératoire après les interventions de décompression et pour l'évaluation de l'efficacité de certains traitements de l'ostéopétrose [24,25,74].

\subsection{Imagerie par résonance magné- tique (IRM)}

L'IRM est principalement utilisée dans l'ostéopétrose comme un indicateur de l'activité de la moelle osseuse. La forme maligne se caractérise par une absence complète de signal pour les corps vertébraux qui apparaissent «noirs » et contrastent de ce fait fortement avec les disques médecine

buccale

chirurgie

buccale

VOL. $12, \mathrm{~N}^{\circ} 3$ 2006

page 144 intervertébraux adjacents. L'IRM semble de plus très utile dans l'évaluation de l'efficacité de la transplantation de moelle osseuse en montrant la recolonisation des cavités médullaires par le

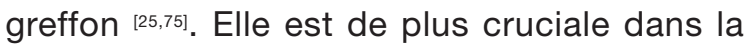
recherche d'une dégénérescence de la substance blanche cérébrale pour l'ostéopétrose associée à une maladie de stockage neuronal [24].

\subsection{Scintigraphie osseuse}

Les indications de la scintigraphie ne diffèrent pas des indications habituelles. Elle se révèle utile pour confirmer le diagnostic d'une ostéomyélite ou d'une fracture occulte qui peut être masquée par l'augmentation significative de la densité osseuse en radiologie conventionnelle ${ }^{[24,25,76]}$. La réalisation d'études spécifiques du système réticulo-endothélial à l'aide de microsphères d'albumine marquées au Tc-99 peut par ailleurs révéler une absence quasi complète d'activité de la moelle osseuse et une hypercaptation dans le foie et la rate, reflétant l'hématopoïèse extramédullaire responsable de l'hépatosplénomégalie [24]. Toutefois, en raison de son faible pouvoir de résolution, la scintigraphie osseuse n'est pas réalisée de routine chez les patients atteints d'ostéopétrose ${ }^{[76]}$.

\subsection{Minéralométrie osseuse}

L'intérêt de la minéralométrie osseuse apparaît secondaire. Elle permet d'une part le contrôle de la masse osseuse dans le cadre de l'évolution naturelle de la sclérose osseuse et, d'autre part, d'évaluer l'effet de la transplantation de moelle sur les os [77]. Cette technique paraît néanmoins décevante dans la détection des porteurs sains de l'ostéopétrose bénigne de type II [78].

\section{EXAMENS DE LABORATOIRE}

\subsection{Manifestations hématologiques}

La forme maligne comporte de nombreuses anomalies : une anémie myélophtisique consécutive au comblement des cavités médullaires et à l'étouffement de la moelle hématopoïétique, une granulopénie et une thrombopénie [25,32,79]. II en résulte une hématopoïèse extramédullaire entraînant une hypertrophie secondaire du foie et de la rate; l'érythropoïèse de stress se traduit par la présence d'hémoglobine fœtale ${ }^{[25]}$. Dans les premières phases de la maladie, la formule sanguine reste sensiblement normale mais témoigne de la réaction érythro-leucocytaire. Avec le développement de l'hépatosplénomégalie, la pancytopénie devient évidente [25].

Dans l'ostéopétrose bénigne il n'y a en général aucune anomalie de l'hémogramme [25,26].

Le déficit en AC II peut s'accompagner d'une discrète anémie, le plus souvent secondaire à une carence alimentaire ${ }^{[24]}$.

\subsection{Chimie sanguine}

Dans la forme maligne, le taux sérique du calcium, des phosphates et de la phosphatase alcaline est en général normal [25]. La calcémie reflète l'apport alimentaire de calcium, et une hypocalcémie fait

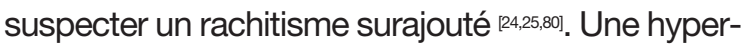
parathyroïdie secondaire, avec un taux élevé de calcitriol, est fréquemment observée ${ }^{[24,25,80]}$; le taux sérique des phosphatases acides est souvent augmenté dans les formes sévères et dans la forme bénigne de type II ${ }^{39,49]}$. Cette augmentation est probablement secondaire au nombre accru d'ostéoclastes, mais sans augmentation concomitante de la résorption osseuse comme semblent le montrer l'expérimentation animale ${ }^{[54,81]}$. En cas de transplantation de moelle osseuse, il n'est pas rare de constater une hypercalcémie sévère secondaire au rétablissement de la fonction ostéoclastique ${ }^{[82]}$. 
Dans la forme bénigne, les valeurs sériques des indices de l'homéostase minérale sont en général normales ${ }^{[24,25]}$. Toutefois, certaines études récentes signalent une élévation du taux de la PTH et de l'isoenzyme BB «cérébral » de la créatinine-kinase dans l'ostéopétrose bénigne de type II [24]. L'isoenzyme BB étant également présent dans les ostéoclastes [24], l'augmentation de ce dernier reflète non pas une lésion du système

\section{RÉFÉRENCES}

1 - KARSHNER RJ. Oteopetrosis. Am J Radiol 1926 ; 16 : 405-19.

2 - AlbeRS-SchÖnBERG H. Röntgenbilder einer seltenen Knochenerkrankung. Münsch Med Wsch 1904 ; 51, 365-9.

3 - Loria-Cortez R, Quesada-Calvo E, Cordero-Chaverri C. Osteopetrosis in children. A report of 26 cases. J Pediatr $1977 ; 91: 43-7$.

4 - WhYTE MP. Osteopetrosis (pp 789-807). In: Royce PM, Steinmann B, eds. Connectivetissue and its heritable disorders. Molecular, genetic and medical aspects. Wiley-Liss, New-York, 2002.

5 - Bollerslev J. Osteopetrosis. A genetic and epidemiological study. Clin Genet 1987 ; 31 : 86-90.

6 - Guibaud P, Larbre F, Freycon MT, Genoud J. Osteopetrosis and renal tubular acidosis. 2 cases of this association in a sibship. Arch Fr Pediatr 1972 ; 29 : 26986.

7 - Sly WS, LANG R, Avioli L, Haddad J, Lubowitz H, Mc Alister W. Recessive osteopetrosis: new clinical phenotype. Am J Hum Genet 1972 ; 24 : 34.

8 - Vainsel M, Fondu P, Cadranel S, Rocmans C, Gepts W. Osteopetrosis associated with proximal and distal tubular acidosis. Acta Paediatr Scand 1972 ; 61 : 429-34.

9 - Whyte MP, MuRPhy WA, FALLON MD, SLY WS, Teitelbaum SL, McAlister WH, Aviol LV. Osteopetrosis, renal tubular acidosis and basal ganglia calcification in three sisters. Am J Med 1980 ; 69 : 64-74.

10 - Sly WS, HeWett-EMmett D, Whyte MP, Yu YS, TASHIAN RE. Carbonic anhydrase II deficiency identified as the primary defect in the autosomal recessive syndrome of osteopetrosis with renal tubular acidosis and cerebral calcification. Proc Natl Acad Sci USA 1983 ; $80: 2752-6$

11 - Beighton P, Hamersma H, Cremin BJ. Osteopetrosis in South Africa. The benign, lethal and intermediate forms. S Afr Med J 1979 ; 55 : 659-65.

12 - Kahler SG, Burns JA, AyLSWORTh AS. A mild autosomal recessive form of osteopetrosis. Am J Med Genet $1984 ; 17: 451-64$.

13 - Horton WA, Schimke RN, IyAma T. Osteopetrosis: further heterogeneity. J Pediatr 1980 ; 97 : 580-5. nerveux central mais une activation des ostéoclastes ou des cellules résorbantes immatures au sein de l'os sclérotique hypoxique, où la créatinine-phosphate est utilisée comme principale source d'énergie anaérobie ${ }^{[24,83]}$.

Le déficit en AC II peut s'accompagner d'une acidose métabolique dans la période néonatale et d'une acidose tubulaire rénale de type I et II [24,84].

14 - El Khazen N, Faverly D, Vamos E, Flament-Durand J, Carton B, Cremer-Perlmutter N. Lethal osteopetrosis with multiple fractures in utero. Am J Med Genet 1986 ; $23: 811-9$.

15 - Jagadha V, Halliday WC, Becker LE, Hinton D. The association of infantile osteopetrosis and neuronal storage disease in two brothers. Acta Neuropathol 1988 ; $75: 233-40$.

16 - Monaghan BA, Kaplan FS, August CS, Fallon MD, FLANNERY DB. Transient infantile osteopetrosis. J Pediatr $1991 ; 118: 252-6$.

17 - Labat ML, Bringuier aF, Chandra A, EInhorn TA, CHANDRA P. Retroviral expression in mononuclear blood cells isolated from a patient with osteopetrosis (AlbersSchonberg disease). J Bone Miner Res 1990 ; 5 : 42535.

18 - MiLls BG, YABE H, Singer FR. Osteoclasts in human osteopetrosis contain viral-nucleocapsid-like nuclear inclusions. J Bone Miner Res 1988 ; 3 : 101-6.

19 - Kovacs CS, Lambert RG, Lavole GJ, SiminoskI K. Centrifugal osteopetrosis: appendicular sclerosis with relative sparing of the vertebrae. Skeletal Radiol 1995 ; $24: 27-9$.

20 - Whyte MP, Wenkert D, Clements KL, McAlister WH, Mumm S. Bisphosphonate-induced osteopetrosis. N Engl J Med 2003 ; 349 : 457-63.

21 - Dudin AA, Rambaud-Cousson A. Syndrome of infantile osteopetrosis and Hirschsprung disease in seven children born to four consanguineous unions in two families. Am J Med Genet 1993 ; 47 : 1083-5.

22 - Migliaccio S, Luciani M, Taranta A, , Minisola S, El Hachem M, Bosman C, De Felice L, Boldrini R, Corsi A, BIANCO P, TETI A. Association of intermediate osteopetrosis with poikiloderma. J Bone Miner Res $1999 ; 14: 834-6$.

23 - Dupuis-Girod S, Corradini N, Hadj-Rabia S, Fournet JC, Faivre L, Le Deist F, Durand P, Doffinger R, Smah A, Israel A, Courtois G, Brousse N, Blanche S, Munnich A, Fischer A, Casanova JL, Bodemer C. Osteopetrosis, lymphedema, anhidrotic ectodermal dysplasia, and immunodeficiency in a boy and incontinentia pigmenti in his mother. Pediatrics $2002 ; 109: 97$. \begin{tabular}{l}
$\begin{array}{l}\text { médecine } \\
\text { buccale } \\
\text { chirurgie } \\
\text { buccale }\end{array}$ \\
\hline VOL. $12, N^{\circ} 3$ \\
2006
\end{tabular}

page 145 
24 - WHYTE MP. Sclerosing bone disorders (pp 789-807). In: Research ASfBaM, ed. Primer on the metabolic bone diseases and disorders of mineral metabolism, 5th ed. American society for bone and mineral research, Washington, 2003.

25 - ShAPIRO F. Osteopetrosis. Current clinical considerations. Clin Orthop 1993 ; 34-44.

26 - Bollerslev J, MoseKILDE L. Autosomal dominant osteopetrosis. Clin Orthop 1993 ; 45-51.

27 - RUBEN JB, MorRIS RJ, JUDISCH GF. Chorioretinal degeneration in infantile malignant osteopetrosis. Am J Ophthalmol $1990 ; 110: 1-5$.

28 - Lehman RA, Reeves JD, Wilson WB, Wesenberg RL. Neurological complications of infantile osteopetrosis. Ann Neurol $1977 ; 2$ : 378-84.

29 - JOHnston CCJ, LAVY N, LORD T, Vellios F, MerRitT AD, DEISS WP, JR. Osteopetrosis. A clinical, genetic, metabolic, and morphologic study of the dominantly inherited, benign form. Medicine (Baltimore) 1968 ; 47 : 149-67.

30 - Beard CJ, Key L, Newburger PE, Ezekowitz RA, Arcecl R, Miller B, Proto P, Ryan T, Anast C, SIMONS ER. Neutrophil defect associated with malignant infantile osteopetrosis. J Lab Clin Med 1986 ; 108 : 498505.

31 - Banco R, Seifert MF, Marks SC, JR., McGuire JL. Rickets and osteopetrosis: the osteosclerotic (oc) mouse. Clin Orthop 1985 : 238-46.

32 - McCUNE D. Osteopetrosis (marble bone) in an infant. Review of the litterature and report of a case. Am J Dis Child $1943 ; 48$ : 949-955.

33 - Milhaud G, LABAt ML, LitWin I, Moricard Y, Moutier R, Rimbaut C, BufFe D, Juster M. Osteopetro-rickets: a new congenital bone disorder. Metab Bone Dis Relat Res $1981 ; 3: 91-7$.

34 - DoRANTES LM, MEJIA AM, Dorantes S. Juvenile osteopetrosis: effects on blood and bone of prednisone and a low calcium, high phosphate diet. Arch Dis Child $1986 ; 61: 666-70$.

35 - ReEves J, ARnaud S, Gordon S, et AL. The pathogenesis of infantile malignant osteopetrosis: bone mineral metabolism and complications in five infants. Metab Bone Dis Relat Res 1981 ; 3 : 135-42.

36 - Teltelbaum SL, Coccia PF, Brown DM, Kahn AJ. Malignant osteopetrosis: a disease of abnormal osteoclast proliferation. Metab Bone Dis Relat Res 1981 ; 3 : 99-105.

37 - O'Meara A, Breatnach F, Kelleher J. Misleading radiology following bone marrow transplantation for infantile osteopetrosis. Eur J Pediatr 1990 ; 149 : 447-8.

38 - BOLLERSLEV J. Autosomal dominant osteopetrosis: bone metabolism and epidemiological, clinical, and hormonal aspects. Endocr Rev 1989 ; 10 : 45-67.

39 - Bollerslev J, Andersen PE JR. Radiological, biochemical and hereditary evidence of two types of autosomal dominant osteopetrosis. Bone $1988 ; 9: 7-13$.
40 - Walpole IR, Nicoll A, Goldblatt J. Autosomal dominant osteopetrosis type II with "malignant" presentation: further support for heterogeneity? Clin Genet 1990 ; $38: 257-63$

41 - Andersen PE JR, BollersLeV J. Heterogeneity of autosomal dominant osteopetrosis. Radiology 1987 ; 164 : 223-5.

42 - TriAs A, Fery A. Ostéopétrose chez l'adulte. Rev Chir Orthop Reparatrice Appar Mot 1974 ; 60 : 593-606.

43 - BOLLERSLEV J, ANDERSEN PE JR. Fracture patterns in two types of autosomal-dominant osteopetrosis. Acta Orthop Scand 1989 ; 60 : 110-2.

44 - Bollerslev J, Grodum E, Grontved A. Autosomal dominant osteopetrosis (a family study). J Laryngol Otol 1987 ; 101 : 1088-91.

45 - KleInBERg S. Osteopetrosis. Am J Surg 1954 ; 87 : 5062.

46 - MILGRAM JW, JASTY M. Osteopetrosis. A morphological study of twenty-one cases. J Bone Joint Surg Am 1982;64:912-29.

47 - Beighton P, Horan F, Hamersma H. A review of the osteopetrosis. Postgrad Med J 1977 ; 53 : 507-16.

48 - KLINWORTH GK. The neurological manifestations of osteopetrosis (Albers-Schönberg) disease. Neurology $1963 ; 13: 512-9$.

49 - Shapiro F, Glimcher MJ, HOLtrop ME, TASHJIAN AH JR, Brickley-PARSOnS D, KenZORA JE. Human osteopetrosis: a histological, ultrastructural, and biochemical study. J Bone Joint Surg Am 1980 ; 62 : 384-99.

50 - YARINGTON CT JR, SPRINKLE PM. Facial palsy in osteopetrosis. Relief by endotemporal decompression. JAMA $1967 ; 202: 549$.

51 - CAMERON HU, DeWAR FP. Degenerative osteoarthritis associated with osteopetrosis. Clin Orthop 1977 ; 148-9.

52 - MAZUR J, WORTSMAN J. Hypogonadotropic hypogonadism from osteopetrosis. Clin Orthop 1982 ; 202-6.

53 - SLY WS, SHAH GN. The carbonic anhydrase II deficiency syndrome: Osteopetrosis with renal tubular acidosis and cerebral calcification (pp: 5331-43). In: Scriver CR, Beaudet AL, Sly WS, Valle D, eds. The metabolic and molecular bases of inherited disease, McGraw-Hill, New-York, 2001.

54 - WHYTE MP. Carbonic anhydrase II deficiency. Clin Orthop 1993 : 52-63.

55 - Roth DE, Venta PJ, TAShian RE, SLy WS. Molecular basis of human carbonic anhydrase II deficiency. Proc Natl Acad Sci USA 1992 ; 89 : 1804-8.

56 - Sly WS, Whyte MP, Sundaram V, TASHIAN RE, HEWEtTEmmett D, Guibaud P, Vainsel M, Baluarte hJ, Gruskin A, Al-Mosawi M. Carbonic anhydrase II deficiency in 12 families with the autosomal recessive syndrome of osteopetrosis with renal tubular acidosis and cerebral calcification. N Engl J Med 1985 ; 313 : 139-45.

57 - Ohlsson A, Cumming WA, PAUl A, SLy WS. Carbonic anhydrase II deficiency syndrome: recessive osteopetrosis with renal tubular acidosis and cerebral calcification. Pediatrics $1986 ; 77: 371-81$. 
58 - Venta PJ, Welty RJ, Johnson tM, Sly WS, TAShian RE. Carbonic anhydrase II deficiency syndrome in a Belgian family is caused by a point mutation at an invariant histidine residue (107 His----Tyr): complete structure of the normal human CA II gene. Am J Hum Genet 1991 ; 49 : 1082-90.

59 - Bejaoui M, Kamoun A, Baraket M, Bourguiba $H$, LAKHOUA R. Syndrome associating osteopetrosis, tubular acidosis, mental retardation and cerebral calcifications due to carbonic anhydrase II deficiency. A propos of 3 cases in the siblings. Arch Fr Pediatr 1991; 48 : 211-4.

60 - Cochat P, Guibaud P, Sly WS, LaRbre F. Osteopetrosis, renal tubular acidosis and cerebral calcifications: Followup of 3 children in 2 untreated families. Helv Paediatr Acta $1986 ; 41: 156$.

61 - Cochat P, LoRas-Duclaux I, Guibaud P. Carbonic anhyDRASE II DEFICIENCY: OSTEOPETROSIS, RENAl tubular acidosis and intracranial calcifications. Review of the literature and 3 cases. Pediatrie $1987 ; 42: 121-8$.

62 - Tashian RE, Hewett-Emmett D, Goodman M. On the evolution and genetics of carbonic anhydrase I, II and III (pp 79-100). In: Rattazzi ME, Mario C, Scansalios JG, Whitt GS, eds. Isoenzymes: current topics in biological and medical research, Alan R. Liss, New York, 1983.

63 - Dodgson SJ, Forster RE, 2ND, SLY WS, TASHIAN RE. Carbonic anhydrase activity of intact carbonic anhydrase II-deficient human erythrocytes. J Appl Physiol $1988 ; 65: 1472-80$.

64 - Strisciuglio P, Sartorio R, Pecoraro C, Lotito F, SLY WS. Variable clinical presentation of carbonic anhydrase deficiency: evidence for heterogeneity? Eur J Pediatr $1990 ; 149$ : 337-40.

65 - Al Rajeh S, El Mouzan Mi, Ahlberg A, Ozaksoy D. The syndrome of osteopetrosis, renal acidosis and cerebral calcification in two sisters. Neuropediatrics 1988 ; 19 : 162-5.

66 - LABAT ML. Retroviruses and bone diseases. Clin Orthop 1996 ; 287-309.

67 - PeRBAL B. Pathogenic potential of myeloblastosis-associated viruses. Infect Agents Dis 1995 ; 4 : 212-27.

68 - EISENBERG DL. Clinical Imaging: an atlas of differential diagnosis (pp 375-84), 3rd ed. Lippincott-Raven, Philadelphia, 1997.

69 - Kolawole tM, Hawass nd, Patel PJ, Mahd AH. Osteopetrosis: some unusual radiological features with a short review. Eur J Radiol 1988 ; 8 : 89-95.

70 - HINKEL CL, BEILER DD. Osteopetrosis in adults. Am J Roentgenol Radium Ther Nucl Med 1955 ; 74 : 46-64.

71 - Paupe J, Nezeloff C, Denan B, Vialatte J. Osteopetrosis with rachitism. A propos of a case with anatomopathological studies. Ann Pediatr (Paris) 1967 ; 14 : 2451-61.

72 - HoRAN F, BEIGHTON P. Osteopetrosis in the fairbank collection. J Bone Joint Surg Am 1978 ; 60B : 53-5.

73 - Cumming WA, OHLSSON A. Intracranial calcification in children with osteopetrosis caused by carbonic anhydrase II deficiency. Radiology 1985 ; 157 : 325-7.
74 - Al-Mefty O, Fox JL, Al-Rodhan N, Dew JH. Optic nerve decompression in osteopetrosis. J Neurosurg 1988 ; $68: 80-4$

75 - RaO VM, Dalinka MK, Mitchell DG, Spritzer CE, Kaplan F, August CS, axel L, Kressel hy. Osteopetrosis: MR characteristics at 1.5 T. Radiology $1986 ; 161: 217-20$.

76 - AdAms BK. Scintigraphy in a patient with complicated osteopetrosis. Clin Nucl Med 1989; 14 : 323-6.

77 - Kaplan FS, August CS, Dalinka MK, Karp J, Fallon MD, HADDAD JG. Bone densitometry observations of osteopetrosis in response to bone marrow transplantation. Clin Orthop 1993 ; 79-84.

78 - TAKAcs I, COOPER H, WeAVER DD, ECONs MJ. Bone mineral density and laboratory evaluation of a type II autosomal dominant osteopetrosis carrier. Am J Med Genet $1999 ; 85: 9-12$.

79 - PINES B, LeDERER M. Osteopetrosis: Albers-Schönberg disease (marble bone). Report of case and morphologic study. Am J Pathol 1947 ; 23 : 755-75.

80 - Cournot G, Trubert-Thil CL, Petrovic M, TrubertThil Cl, Petrovic M, Boyle A, Cormier C, Girault D, FISCHER A, GaRABEDIAN M. Mineral metabolism in infants with malignant osteopetrosis: heterogeneity in plasma 1,25-dihydroxyvitamin D levels and bone histology. J Bone Miner Res 1992 ; 7 : 1-10.

81 - SEIFERT MF. The biology of macrophages in osteopetrosis. Structure and function. Clin Orthop 1984 ; 270-7.

82 - Rawlinson PS, Green RH, Coggins AM, Boyle IT, GIBSON BE. Malignant osteopetrosis: hypercalcaemia after bone marrow transplantation. Arch Dis Child 1991 ; $66: 638-9$.

83 - Gram J, Antonsen S, Horder M, Bollerslev J. Elevated serum levels of creatine kinase BB in autosomal dominant osteopetrosis type II. Calcif Tissue Int 1991 ; 48 : 438-9.

84 - SLY WS, Whyte MP, KRUPIN T, SUndaRAm V. Positive renal response to intravenous acetazolamide in patients with carbonic anhydrase II deficiency. Pediatr Res 1985 ; $19: 1033-6$.

85 - Baron R. General principles of bone biology (pp 1-8). In: Favus MJ, ed. Primer on the metabolic bone disease and disorders of mineral metabolism, 5th ed. American society for bone and mineral research, Washington, 2003.

86 - Schenk RK, Hofstetter W, Felix R. Morphology and chemical composition of connective tissue: bone (pp 67-120). In: Royce PM, Steinmann B, eds. Connective tissue and its heritable disorders. Molecular, genetic and medical aspects, Wiley-Liss, New York, 2002.

87 - SolCia E, Rondini G, CAPELLA C. Clinical and pathological observations on a case of newborn osteopetrosis. Helv Paediatr Acta 1968 ; 23 : 650-8.

88 - HASENHUTTL K. Osteopetrosis. Review of the literature and comparative studies on a case with a twenty-fouryear follow-up. Am J Orthop 1962 ; 44-A : 359-70. \begin{tabular}{l}
$\begin{array}{l}\text { médecine } \\
\text { buccale } \\
\text { chirurgie } \\
\text { buccale }\end{array}$ \\
\hline vOL. $12, N^{\circ} 3$ \\
2006 \\
\hline page 147
\end{tabular} 
89 - Ruprecht A, WAgneR H, ENGEL H. Osteopetrosis: report of a case and discussion of the differential diagnosis. Oral Surg Oral Med Oral Pathol 1988 ; 66 : 674-9.

90 - BRECK LW, CORNELL RC, EMMETT JE. Intramedullary fixation of fractures of the femur in a case of osteopetrosis. J Bone Joint Surg Am 1957 ; 39-A : 1389-94.

91 - Lawoyin DO, Daramola JO, Ajagbe HA, Nyako EA, LAWOYIN JO. Osteomyelitis of the mandible associated with osteopetrosis: report of a case. Br J Oral Maxillofac Surg $1988 ; 26: 330-5$

92 - Blin-WaKKach C, Bernard F, CARLE GF. Osteopetrosis, from mouse to man. Med Sci $2004 ; 20: 61-7$.

93 - Mundy GR, Chen D, OYAJOBI O. Bone remodelling (pp 46-58). In: Favus MJ, ed. Primer on the metabolic bone diseases and disorders of mineral metabolism, 5th ed. American society for bone and mineral research, Washington, 1993.

94 - Malkani K, Luxembourger MM, Rebel A. Cytoplasmic modifications at the contact zone of osteoclasts and calcified tissue in the diaphyseal growing plate of foetal guinea-pig tibia. Calcif Tissue Res 1973 ; 11 : 25864.

95 - KING GJ, HoLtROP ME. Actin-like filaments in bone cells of cultured mouse calvaria as demonstrated by binding to heavy meromyosin. J Cell Biol $1975 ; 66$ : 445-51.

96 - Felix R, Hofstetter W, CeCChINI MG. Recent developments in the understanding of the pathophysiology of osteopetrosis. Eur J Endocrinol 1996 ; 134 : 143-56.

VOL. $12, \mathrm{~N}^{\circ} 3$ 2006

page 148
104 - Coccia PF, Krivit W, Cervenka J, Clawson C, Kersey JH, Kim TH, Nesbit ME, Ramsay NK, Warkentin PI, Teitelbaum SL, Kahn AJ, Brown DM. Successful bone-marrow transplantation for infantile malignant osteopetrosis. N Engl J Med 1980 ; 302 : 701-8.

105 - Kaplan FS, August CS, Fallon MD, Dalinka M, Axel L, HADDAD JG. Successful treatment of infantile malignant osteopetrosis by bone-marrow transplantation. A case report. J Bone Joint Surg Am 1988 ; 70 : 617-23.

106 - WHYTE MP. Chipping away at marble-bone disease. N Engl J Med 1995 ; 332 : 1639-40.

107 - Teitelbaum SL, Tondravi MM, Ross FP. Osteoclast biology (pp 61-94). In: Marcus R, Feldman D, Kelsey D, eds. Osteoporosis, Academic Press, San Diego, 1996.

108 - MARKS SC JR. Osteopetrosis: multiple pathways for the interception of osteoclast function. Appl Pathol $1987 ; 5: 172-83$.

109 - KEY LL JR, ROdRIGUIZ RM, WILLI SM, WRIGHT NM, Hatcher HC, Eyre DR, CuRE JK, GrifFIn PP, RIES WL. Long-term treatment of osteopetrosis with recombinant human interferon gamma. N Engl J Med 1995 ; 332 : 1594-9.

110 - BolleRsLeV J, KVEtNy J. Thyroid hormone resistance in blood monocyte cells and elevated serum T3 in patients with autosomal dominant osteopetrosis. Scand J Clin Lab Invest 1988 ; 48 : 795-9.

111 - KEY LL, RIES WL, SCHIFF R. Osteopetrosis associated with interleukin-2 deficiency. J Bone Miner Res 1987 ; 2 (suppl. ):87.

112 - Glorieux FH, Pettifor JM, Marie PJ, Delvin Ee, TRAVERS R, SHEPARD N. Induction of bone resorption by parathyroid hormone in congenital malignant osteopetrosis. Metab Bone Dis Relat Res 1981 ; 3 : 143-50.

113 - SCHNEIDER GB, RELFSON M. Effects of interleukin-2 on bone resorption and natural immunity in osteopetrotic (ia) rats. Lymphokine Cytokine Res 1994 ; 13 : 335-41.

114 - FELIX R, CECCHINI MG, FLEISCH H. Macrophage colony stimulating factor restores in vivo bone resorption in the op/op osteopetrotic mouse. Endocrinology 1990 ; $127: 2592-4$.

115 - Key LL, Ries WL, GLASSCOCK HC, Jaffe H. Treatment of human osteopetrosis with recombinant interferongamma. J Bone Miner Res 1990 ; 6 (suppl. 1) : S-192.

116 - SANYAL G, MAREN TH. Thermodynamics of carbonic anhydrase catalysis. A comparison between human isoenzymes B and C. J Biol Chem 1981 ; 256 : 608-12.

Voir la suite des références en page 158 de ce numéro. 